\title{
Fire-Needle Acupuncture Treatment in Scapular Muscle Fasciitis
}

\author{
Jihe Zhu' ${ }^{1}$ Blagica Arsovska1,2, Kristina Kozovska1,3* \\ ${ }^{1}$ Faculty of Medical Sciences, University Goce Delchev, Shtip, Republic of Macedonia \\ ${ }^{2}$ Institute of Biology, Faculty of Natural Sciences and Mathematics, Skopje, Republic of Macedonia \\ ${ }^{3}$ Medicine Faculty, St. Cyril and Methodius University of Skopje, Skopje, Republic of Macedonia \\ Email: *tongdatang-tcm@hotmail.com
}

How to cite this paper: Zhu, J.H., Arsovska, B. and Kozovska, K. (2019) Fire-Needle Acupuncture Treatment in Scapular Muscle Fasciitis. Yangtze Medicine, 3, 283-288. https://doi.org/10.4236/ym.2019.34027

Received: May 19, 2019

Accepted: September 14, 2019

Published: September 17, 2019

Copyright (c) 2019 by author(s) and Scientific Research Publishing Inc. This work is licensed under the Creative Commons Attribution International License (CC BY 4.0).

http://creativecommons.org/licenses/by/4.0/

\begin{abstract}
The scapula (shoulder blade) is a large triangular-shaped bone located in the upper back and includes two muscles on each side. The scapula is surrounded and supported by a complex system of muscles that work together to help in the arm movement. If some condition or injury causes these muscles to become imbalanced or weak, the position of the scapula can be altered at rest or in motion. A change in the scapular positioning or motion can make difficulties in the arm movement, especially when performing overhead activities and may cause shoulder weakness. Acupuncture treatment as part of the Traditional Chinese Medicine is most commonly used treatment for treating musculoskeletal disorders. In the treatment of scapular muscle fasciitis the fire-needle acupuncture gave the fastest and best results in our practice. The aim of the treatment is to break up the scar tissue and the adhesions which commonly form at the site, to relax the levator scapula muscle and restore local normal blood and energy flow. In the research are included 13 patients, 6 male and 7 female, on age from 24 to 70 . All patients had scapular pain and on all was performed fire-needle acupuncture. Most common age groups are 50 - 60, most patients have pain on the right scapula and most of them-9 have done only one treatment. A long-term problem with the scapula muscle can be resolved with only one fire-needle acupuncture treatment.
\end{abstract}

\section{Keywords}

Acupuncture, Traditional Chinese Medicine, Treatment, Scapula, Pain

\section{Introduction}

The scapula (shoulder blade) is a large triangular-shaped bone located in the upper back and includes two muscles on each side. The scapula is surrounded 
and supported by a complex system of muscles that work together to help in the arm movement. If some condition or injury causes these muscles to become imbalanced or weak, the position of the scapula can be altered at rest or in motion. A change in the scapular positioning or motion can make difficulties in the arm movement, especially when performing overhead activities and may cause shoulder weakness. Another medical term used for these alterations is scapular dyskinesis.

Scapular pain may occur on one or on both sides of the body, but most commonly it is located on the one scapula. Symptoms that may occur are: pain and tenderness around the scapula, weakness in the affected arm, limited range of motion, fatigue during repetitive activities especially overhead movements, a "snapping" or "crunching" sound during shoulder movement, noticeable protrusion (winging) of the scapula and other [1].

The Western medicine treatment aims for strengthening the muscles in the shoulder and restoring the proper position and motion of the scapula.

The levator scapula muscle's principle action is to elevate the shoulder blade up and forward. A common (often sub-conscious) physical response to emotional stress is to engage the levator scapula muscle in a self-protective reflex by elevating the shoulder blade to protect the cervical spine. When the stress is chronic, the levator muscle never gets to relax and can develop tight and very painful trigger points or possibly tendonitis at its attachment to the scapula at the site of the acupuncture point SI14 (Jianweishu). Chronic tension in the levator muscle at the same time pulls down and sideways on the cervical spine and causes neck pain and compresses the rotator cuff tendons located under the acromial arch of the shoulder blade, which by the way can also lead to rotator cuff syndrome [2].

Acupuncture treatment as part of the Traditional Chinese Medicine (TCM) is most commonly used treatment for treating musculoskeletal disorders. In the terms of TCM, invasion and overexposure of the external factors-Cold, Wind, Heat and Damp are one of the most common causes of both acute and chronic scapular pain and are core principles in TCM. These factors can block the channels and cause stagnation of the Blood and Qi around the scapular region. Wind usually attacks the upper body in combination with the other pathogenic factors, such as Cold, Damp and Heat.

The Wind-Cold cause contractions of the muscles and tendons, stagnation of the Blood and Qi in the channels and sudden and severe pain in the scapula accompanied with cold sensation.

The Wind-Heat invades the scapular region, causing scapular pain with burning and hot sensation, swelling in the scapular area and limitation of the shoulder movement.

The Wind-Damp disturbs the circulation of the Blood and Qi and causes scapular pain accompanied with swelling and heaviness.

The scapular pain can also be caused by emotional factors like excessive stress, anger and frustration, which cause stagnation in the liver meridians around the 
scapula. Excessive sadness and grief can also disturb the lungs and lead to stagnation in the Lung meridians and cause scapular pain.

In the treatment of scapular muscle fasciitis the fire-needle acupuncture gave the fastest and best results in our practice. The aim of the treatment is to break up the scar tissue and the adhesions which commonly form at the site, to relax the levator scapula muscle and restore local normal blood and energy flow.

\section{Material and Methods}

In the research are included 13 patients, 6 male and 7 female, on age from 24 to 70. All patients had scapular pain and on all of them was performed fire-needle acupuncture. Treatments were done in a clinic for TCM and acupuncture in Skopje, Macedonia by a doctor specialist in acupuncture in a period of one year March 2018 - March 2019. Treatments were done indoor, on a room temperature, with duration of 5 - 10 minutes.

In the treatment were included patients who have finished the treatments, patients who signed an agreement, patients with scapular pain and patients without medication therapy.

In the treatment were not included patients who have not agreed on signing ethical clearance, patients who haven't finished the treatments, children, pregnant women, patients older than 70 years, mentally not stable patients, alcoholics and patients with weak constitution.

Fire-needle acupuncture is an acupuncture technique which involves quick insertion of a heated needle into the area on the body that needs to be treated. In the treatment was treated only one trigger point located on the scapula. The point was needled approximately 10 times with fire-needle acupuncture. The points were chosen because all patients confirmed pain in that area and all had inflammation in the scapular muscle fasciitis. The treated point and the performing of the needling are shown in Figures 1-3.

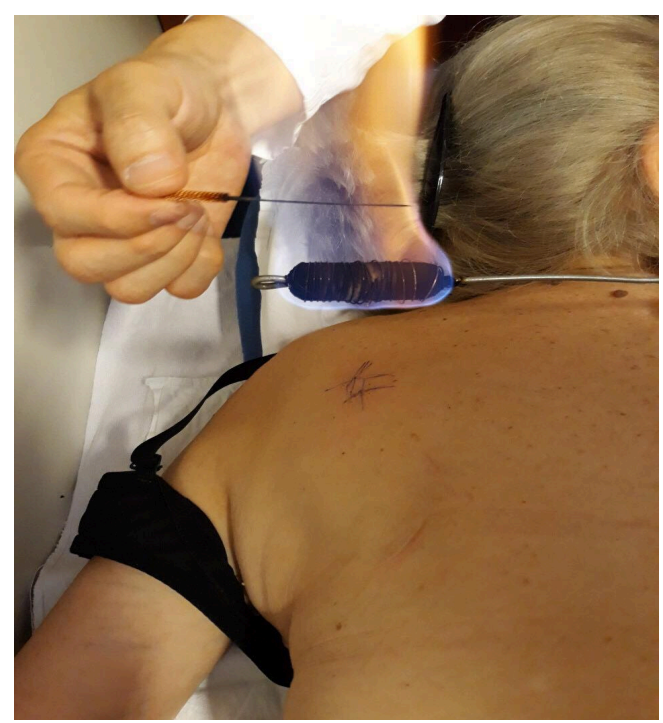

Figure 1. Warming the fire needle before needling into the skin. 


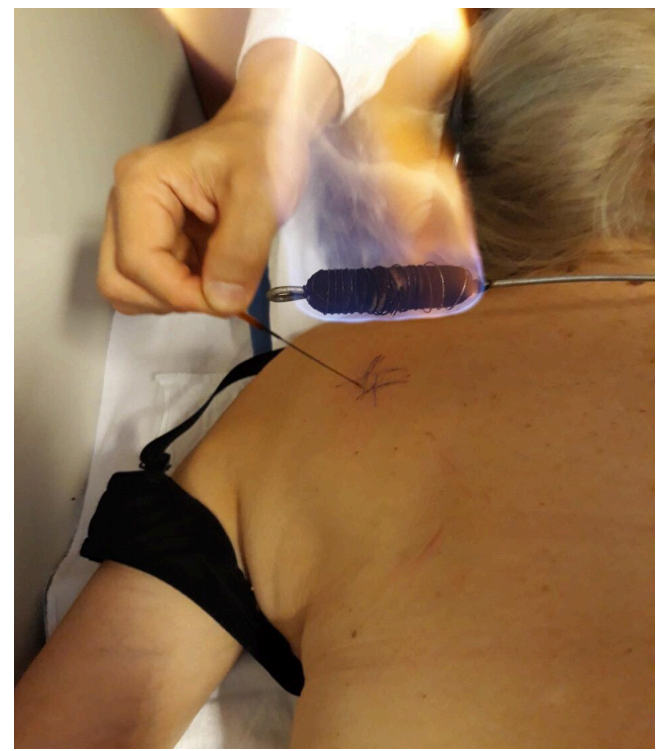

Figure 2. Performing the fire-needle treatment on the trigger point.

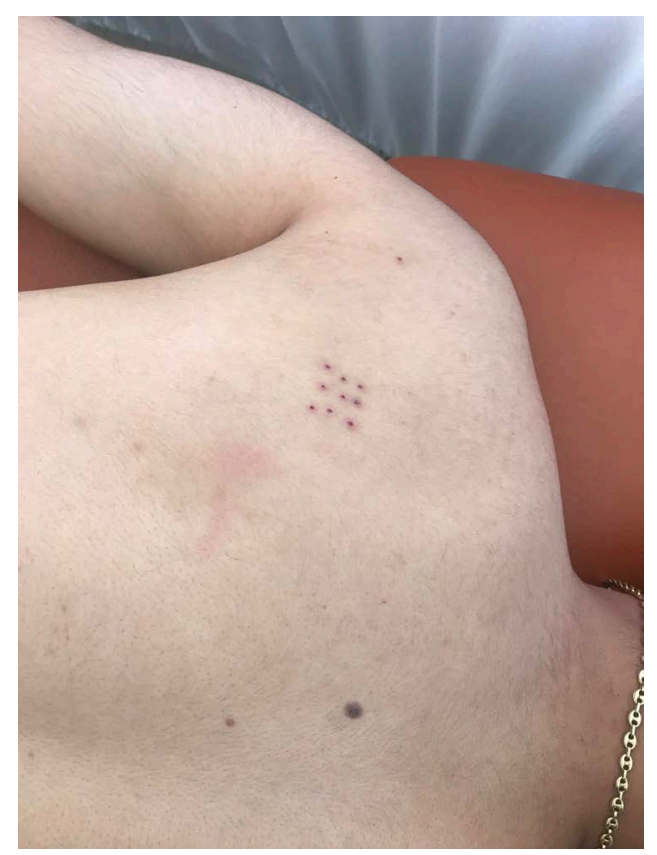

Figure 3. Treated trigger point after the fire-needle treatment.

All patients have signed a consent for ethical clearance.

\section{Results and Discussion}

In the research were included patients who have already finished their treatments. 13 patients were included, 6 male and 7 female. The patients were on age from 24 to 70. The patients were divided into 6 age groups of which most common age groups is $50-60-5$ patients. The results are shown in Table 1.

In all the patients the pain was located only on the one scapular region. 6 patients had pain in the left scapula and 7 in the right. Most of the patients-9 have 
done only one treatment. 3 patients have done 2 treatments and 1 patient have done 3 treatments. The same results are shown in Table 2. After the treatments the patients were free of the pain and all the symptoms were gone permanently.

For assessment of the patient's pain was used numeric rating scale (NRS) shown in Table 3.

Before starting the treatment all patients stated that have severe pain, 7 - 10 on the scale. After the treatments the pain was totally gone in all patients i.e. 0 on the scale (no pain).

Muscular-skeletal disorders are one of the most common problems treated by acupuncture. However, these disorders in which are involved the muscles, bones, tendons, joints and ligaments can be much more deeply rooted and complex than they appear to be. Sometimes there is no clear cause to the problem, the patient may not had an injury or trauma to the affected area. The pain can appear one day out of nowhere. No matter what symptoms are presented clinically, the patient's pulse should always be checked, because the symptoms may come from many aspects of the mind and body and many times they come from places the doctor wouldn't initially suspect.

If there is an injury, then the goal is to repair and heal that injury. Sometimes the process includes just physical reparation. For other patients it may require mental and emotional rebalancing, because traumas can have widespread effects

Table 1. Age groups.

\begin{tabular}{cc}
\hline Age groups & Number of patients \\
\hline $20-30$ & 2 \\
$30-40$ & 1 \\
$40-50$ & 4 \\
$50-60$ & 5 \\
$60-70$ & $/$ \\
$>70$ & 1 \\
\hline
\end{tabular}

Table 2. Number of treatments.

\begin{tabular}{cc}
\hline Number of treatments & Number of patients \\
\hline 1 & 9 \\
2 & 3 \\
3 & 1 \\
\hline
\end{tabular}

Table 3. Numeric rating scale.

\begin{tabular}{cl}
\hline 0 & No Pain \\
$1-3$ & Mild Pain (nagging, annoying, interfering little with daily activities) \\
$4-6$ & Moderate Pain (interferes significantly with daily activities) \\
$7-10$ & Severe Pain (disabling; unable to perform daily activities)
\end{tabular}


on the spirit, body and mind. The human body has an extraordinary ability to store and hide toxins, stress and the trauma effects often in the joints, bones and the blood vessels. This is the reason why many conditions and symptoms require more time, patience and investigation to be uncovered and resolved [3].

In the treatment was used one Ashi or trigger point in all patients. The aim of the filiform fire-needle acupuncture is to transmit the heat into the body at the specific points. Other points that may be used in the treatment are GB-20 (Fengchi), TE-5 (Waiguan), LI-4 (Hegu), SI-4 (Wangu), SI-2 (Tianzong), SI-10 (Naoshu) and BL-58 (Feiyang). On these points is used the twirling reducing method. The method is performed by rotating the needle with the thumb heavily backward and gently forward, counter clockwise for 360 degrees and then clockwise for 360 degrees, sixty times per minute. These points are very good for relieving the back pain and for promoting the circulation of Qi and Blood in the meridians [4].

The main purpose of the treatment is to eliminate the Wind and Cold, dry the Dampness, circulate and harmonize the collaterals, calm and relieve the generalized body pain, relieve the scapular pain, promote the circulation, eliminate the stasis in the Blood in the meridians, warms the body and channels and other. When the external factors, Wind-Cold and the Dampness are eliminated, the circulation of the Blood is regulated, the collaterals are harmonized and the scapular pain is disappearing gradually.

\section{Conclusion}

In the treatment of scapular muscle fasciitis the fire-needle acupuncture gave the fastest and best results in our practice. A long-term problem with the scapula muscle can be resolved with only one fire-needle acupuncture treatment.

\section{Conflicts of Interest}

The authors declare no conflicts of interest regarding the publication of this paper.

\section{References}

[1] Sciascia, D.A. and Kibler, B.W. (2019) Scapular (Shoulder Blade) Disorder. http://www.orthoinfo.aaos.org

[2] Santa Cruz Acupuncture Orthopedics and Sports Medicine Clinic (2019) Acupuncture \& The Levator Scapula Muscle. http://www.santacruzacupunctureclinic.com

[3] Sieben, N. (2018) Unbinding the Chest: Soothing Emotionally Induced Shoulder Pain with Acupuncture. http://www.nicholassieben.com

[4] Lu, J., Guo, Y., Guo, C.Q., Shi, X.M., Du, N., Zhao, R.L., Du, W.P., Liang, J., Zhu, S.P. and Chen, H. (2017) Acupuncture with Reinforcing and Reducing Twirling Manipulation Inhibits Hippocampal Neuronal Apoptosis in Spontaneously Hypertensive Rats. Neural Regeneration Research, 12, 770-778.

https://doi.org/10.4103/1673-5374.206648 